Check for updates

Cite this: RSC Adv., 2017, 7, 41384

Received 22nd June 2017

Accepted 17th August 2017

DOI: $10.1039 / \mathrm{c} 7 \mathrm{ra06965k}$

rsc.li/rsc-advances

\section{High performance boronic acid-containing hydrogel for biocompatible continuous glucose monitoring $\dagger$}

\author{
Qian Dou, ${ }^{a}$ Debo Hu, ${ }^{a}$ Hongkai Gao, ${ }^{b}$ Yongmei Zhang, ${ }^{b}$ Ali K. Yetisen, (D) cd \\ Haider Butt, iD e Jing Wang, ${ }^{\text {a }}$ Guangjun Nie*a and Qing Dai (iD *a
}

\begin{abstract}
Rapid and robust hydrogels are essential in realizing continuous glucose monitoring in diabetes monitoring. However, existing hydrogels are limited in satisfying all of the sensory requirements such as detection range, response time, recoverability and biocompatibility. Here, we have developed a surface-initiated polymerization method to chemically immobilize a nano-boronic acid-hydrogel membrane onto a quartz crystal, then used a quartz crystal microbalance (QCM) to achieve real-time monitoring of glucose. The experimental results show that this hydrogel possesses enhanced binding properties to glucose under physiological conditions ( $\mathrm{pH}$ 7.0-7.5) and blood glucose concentration (BGC) (1.1$33.3 \mathrm{mM})$. Moreover, our hydrogel displayed a rapid response time ( 100 s) to glucose, high biocompatibility in vivo through an animal model. The hydrogel has a great potential as a sensitive glucose probe for implantable continuous glucose sensors.
\end{abstract}

\section{Introduction}

Continuous glucose monitoring systems (CGMS) are the most advanced method for the self-management of diabetes. ${ }^{\mathbf{1 - 4}}$ Quartz crystal microbalance (QCM) is a mass-sensitive sensor characterized by its high sensitivity, fast response and good operability. ${ }^{5,6}$ It can achieve continuous monitoring of glucose concentration in a solution by recording the frequency shift of a quartz crystal before and after contact with the solution. ${ }^{7-9}$ The key challenge for a QCM-based detection platform is to develop a highly-sensitive glucose probe for monitoring in patients' subcutaneous tissues, as the glucose concentrations in interstitial fluid can be correlated with blood glucose under steadystate conditions. ${ }^{\mathbf{1 0 , 1 1}}$ Boronic acid derivatives offer stability, durability, and low cost for application in CGMS development. ${ }^{\mathbf{1 2 - 1 6}}$ In particular, boronic acid-containing hydrogels have high sensitivity to glucose and offer biocompatibility, and have become a promising material for dynamic glucose monitoring in vivo. ${ }^{17-21}$ For example, Sugnaux et al. ${ }^{22}$ synthesized glucose-

\footnotetext{
${ }^{a}$ Division of Nanophotonics, CAS Center for Excellence in Nanoscience, National Center for Nanoscience and Technology, Beijing 100190, P. R. China.E-mail: daiq@ nanoctr.cn; Tel: +86-010-82545720

${ }^{b}$ The Armed Police General Hospital, Beijing, 100039, China

${ }^{c}$ Harvard Medical School, Wellman Center for Photomedicine, Massachusetts General Hospital, 65 Landsdowne Street, Cambridge, Massachusetts 02139, USA

${ }^{d}$ Harvard-MIT Division of Health Sciences and Technology, Massachusetts Institute of Technology, Cambridge, Massachusetts 02139, USA

${ }^{e}$ University of Birmingham, Birmingham B15 2TT, UK

$\dagger$ Electronic supplementary information (ESI) available. See DOI: 10.1039/c7ra06965k
}

sensitive polymer brushes with controllable thickness via surface reversible addition-fragmentation chain-transfer (RAFT) polymerization of 3-methacrylamido phenylboronic acid. The detection range was from 0 to $100 \mathrm{mM}$; however, this sensor operated outside the physiological conditions ( $\mathrm{pH} 9.0$ ) in phosphate-buffered saline (PBS) solutions. To enable analysis under physiological $\mathrm{pH}$, Zhang et al. ${ }^{23}$ have reduced the $\mathrm{p} K_{\mathrm{a}}$ value of the hydrogels by adding acrylamide, and constructed a polymerized crystalline colloidal array. This optical sensor detected glucose ranging from 0 to $50 \mathrm{mM}$ at $\mathrm{pH} 7.4$, but had low sensitivity $(10 \mathrm{mM})$, which was not suitable for accurate detection of glucose required by CGMS. Ye et al. ${ }^{24}$ achieved a high sensitivity $(0.5 \mathrm{mM})$ for glucose by optimizing the concentration of the acrylamide monomer, 3-methacrylamido phenylboronic acid, cross-linker $N, N$-methylenebisacrylamido and solvent dimethyl sulfoxide during the hydrogel preparation, but the detection range was limited to 0.10 to $2.50 \mathrm{mM}$. In addition, the response time of currently reported boronic acidcontaining hydrogels are generally longer than $5 \mathrm{~min},{ }^{22-25}$ which is not within the realm of practical real-time monitoring of BGC. Therefore, to realize the potential of boronic acid-containing hydrogel sensors in CGMS, new hydrogel formulations suitable for fast and sensitive detection of glucose under physiological conditions are urgently needed.

In this study, we fabricated an implantable sensor that meets the physiological requirements for glucose detection, and evaluated its performance through QCM detection. The 3-acrylamidophenylboronic acid (3-APBA) is commercially available and widely used for the detection of glucose. But 3-APBA $\left(\mathrm{p} K_{\mathrm{a}}>8\right)$ have a low sensitivity at the $\mathrm{pH}$ of human 
interstitial fluid (7.0-7.5), ${ }^{\mathbf{2 6 , 2 7}}$ we developed a hydrogel system based on 3-APBA as the glucose-sensing component, and with acrylamide as the monomer and $N, N^{\prime}$-methylenebisacrylamide as the crosslinking agent for hydrogel synthesis. The numerous amine groups introduced into the hydrogel composite membrane hence help to reduce its $\mathrm{p} K_{\mathrm{a}}$ value, and enhance the complexation ability and glucose responsivity of the hybrid membrane at physiological $\mathrm{pH} .{ }^{28}$ Molar ratios of monomers to prepare the hydrogel composition were optimized to operate at physiological glucose concentrations in human interstitial fluid (1.1-33.3 mM). Furthermore, since the real-time sensing capacity of the existing hydrogel sensors was primarily limited by the low association rate of hydrogel film with glucose, we employed a surface-initiated polymerization method to chemically immobilize the nanogel onto a quartz crystal, with the thickness of the nanoscale hydrogel film controlled to improve its response time. By tuning the dimensions of the hydrogel network via varying hydrogel formulations and reaction time, the contact between free glucose molecules and hydrogel network can be maximized. This new boronic acid-containing hydrogel sensor detects glucose at $\mathrm{pH}$ 7.0-7.5 within a detection range of 1.1-33.3 $\mathrm{mM}$ (typical physiological BGC) and a rapid response time ( $\sim 100 \mathrm{~s})$ as compared to existing sensors based on boronic acid in the literature. Subcutaneous implantation experiments in rats indicated that the hydrogel has biocompatibility and durability, and is potentially suitable for in vivo glucose monitoring.

\section{Results and discussion}

\section{Synthesis and characterization}

We employed a surface-initiated polymerization method to immobilize boronic acid-containing hydrogels onto quartz crystals (Fig. 1a). First, the Au substrate was modified with double bonds to functionalize the substrate for polymerization. We used (3-aminopropyl)triethoxysilane (APS) to graft amino groups to the crystal and then used the ringopening reaction of the maleic anhydride with the amino groups to graft $\mathrm{C}=\mathrm{C}$ double bonds onto the crystal. The compound of formula which grown on quartz crystal is shown in Fig. 1a(ii) and the pattern in the red box indicates the double bond on the reaction graft. A pre-polymer solution was then pipetted onto the modified electrode surface and spin-coated to form a liquid layer of uniform thickness. Finally, UV-initiated free-radical polymerization was utilized to cure the monomer layer to form a hydrogel layer having a uniform thickness. The molecular structure of the boronic acid-functionalized hydrogel network is shown in Fig. 1a(iii). The positive and negative regions of the actual map of the coated quartz crystal oscillator are shown in Fig. 1a(i). The boronic acid functional groups in the hydrogel exist in two states that are in equilibrium: a negatively-charged dissociative state or an uncharged non-dissociative state in solution. Boronic acid in the non-dissociative state is in a planar triangular configuration which cannot stably bind to glucose. However, the dissociative state it exhibits a tetrahedral molecular configuration and can form a stable five- or six-membered ring (lactone) with glucose. ${ }^{29,30}$ After glucose molecules in solution bind boronic acid groups through cis-diol groups, the equilibrium shifts in favor of the dissociative state, thus generating more boronic acid molecules in the dissociative state, which can bind to more glucose molecules. ${ }^{31}$

The chemical reactions occurring at the quartz crystal surface were characterized by FTIR after each step of the hydrogel film synthesis process (Fig. 1b). Before amination, there was no obvious IR mode in the spectrum of the acidtreated quartz crystal sample; however, after amination, several IR modes emerged, including the $\mathrm{N}-\mathrm{H}$ stretch mode at $3456 \mathrm{~cm}^{-1}$, the stretch modes of saturated $\mathrm{C}-\mathrm{H}$ at $2912 \mathrm{~cm}^{-1}$ and $2850 \mathrm{~cm}^{-1}$, and the $\mathrm{N}-\mathrm{H}$ in-plane bending mode at $1635 \mathrm{~cm}^{-1}$. The presence of these modes verified the completion of the APS reaction. The appearance of the $\mathrm{C}=\mathrm{C}$ stretch mode at $1585 \mathrm{~cm}^{-1}$ and the decrease of the amino IR modes indicated the completion of the maleic anhydride reaction. Finally, the appearance of the $-\mathrm{B}(\mathrm{OH})_{2}$ mode indicated the deposition of the boronic acid hydrogel. Additionally, X-ray photoelectron spectroscopy (XPS) was used to confirm that the boric acid group was covalently bound to the hydrogel backbone. In the high-resolution scanning spectrum of $\mathrm{B} 1 \mathrm{~s}$, the presence of the boric acid derivative was confirmed by the B $1 \mathrm{~s}$ signal at $191.6 \mathrm{eV}$ (Fig. 1c). Furthermore, the thickness of the film is an important parameter that dictates the performance of the hydrogel. Our experiments indicated that the results have a good platform, fast response time, superior recovery with a film thickness of approximately $250 \mathrm{~nm}$ (Fig. 1d(1)-(3) test results are three different parts of coated crystal).

\section{Glucose sensitivity of boronic acid-containing hydrogel}

The glucose sensitivity of the boronic acid-containing hydrogel was experimentally verified by QCM. Fasting blood glucose concentrations are in the range of 3.9 to $6.1 \mathrm{mM}$ for a healthy person. Concentrations below $3.9 \mathrm{mM}$ are observed in hypoglycemic patients, and concentrations above $11.1 \mathrm{mM}$ are present in diabetic patients. Fig. 2a shows that our hydrogel responds to glucose concentrations ranging from 0.0 to $33.3 \mathrm{mM}$. The hydrogel system has a typical response time (the time span during which the QCM frequency shifts reach the limit of $90 \%$ between two successive glucose concentration steps) of $\sim 100 \mathrm{~s}$ (Fig. 2b). Thus, the dynamic range of our hydrogel-based glucose sensor is broad enough to cover the concentration range required by CGMS. When solutions of increasing glucose concentrations were pumped into the flow cell, the fundamental frequency of the quartz-hydrogel complex resonator decreased due to the association of glucose molecules with the hydrogel matrix, reaching the minimum at $33.3 \mathrm{mM}$. In contrast, when solutions of decreasing glucose concentrations were pumped into the flow cell, the fundamental frequency of the quartz-hydrogel complex resonator increased due to the dissociation of glucose molecules from the hydrogel, reaching the maximum at glucose-free solutions.

The association of glucose molecules with boronic acid molecules in the hydrogel matrix is $\mathrm{pH}$ dependent. To verify that the hydrogel may be adapted to the physiological conditions of human interstitial fluid, we conducted detection 


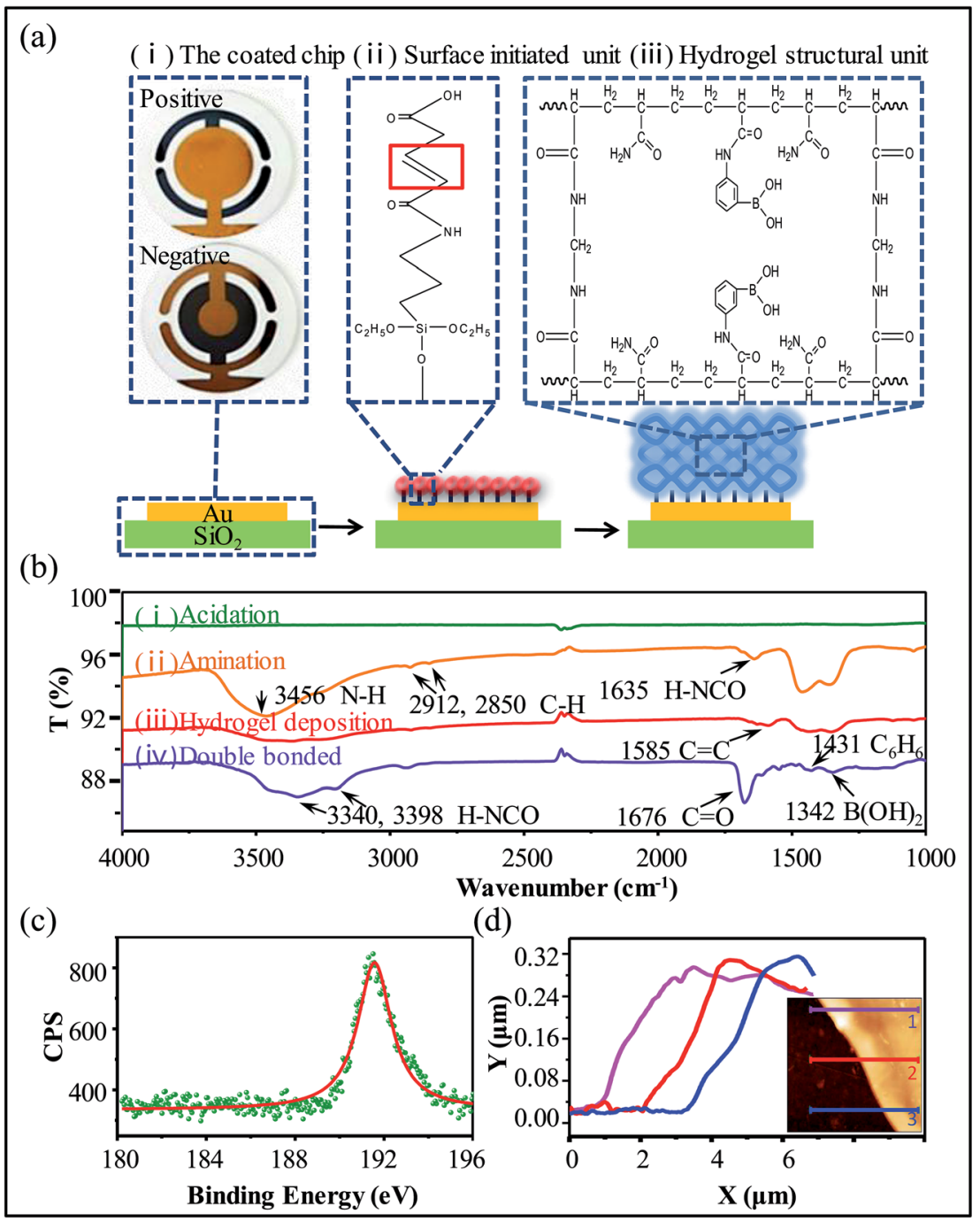

Fig. 1 The synthesis and characterization. (a) Synthesis of the hydrogel-coated quartz crystal. (b) FTIR spectra at different stages of reaction. (c) B 1s XPS spectra of the hydrogel film. (d) Hydrogel thickness characterized by AFM.

experiments with glucose solutions $(0.0-33.3 \mathrm{mM})$ of $\mathrm{pH}$ values ranging from 7.0 to 7.5 (Fig. 2d). The hydrogel was sensitive to glucose and exhibited linearity at different $\mathrm{pH}$ values. Maximum glucose sensitivity was observed at $\mathrm{pH} 7.5$, because higher $\mathrm{pH}$ facilitates the association of glucose molecules with boronic acid molecules in the hydrogel network. Fig. 2c shows that the frequency shift is linearly proportional to the glucose concentration over the range of 0.0-33.3 mM. Deviations from this linear relationship are seen at glucose concentrations above $9.4 \mathrm{mM}$. The larger error at higher glucose concentrations may be due to the conversion between the $1: 1$ binding mode and the $2: 1$ cross-linking mode between the boronic acid and glucose molecules..$^{23,32,33}$ To obtain optimal linear fitting between frequency shift and glucose concentration, we performed the fitting in the ranges of $0.0-9.4 \mathrm{mM}$ and 9.4-33.3 mM. The linear coefficients were $0.9971(\mathrm{pH} \mathrm{7.0)}$, 0.9934 (pH 7.3), and 0.9983 (pH 7.5) in the range of 0 to $9.4 \mathrm{mM}$; and 0.9870 (pH 7.0), 0.9637 (pH 7.3), and 0.9921 (pH 7.5) in the range of 9.4 to $33.3 \mathrm{mM}$. The reversibility of the hydrogel was tested by alternately pumping solutions of low (glucose-free solutions) and high (3.9 $\mathrm{mM})$ glucose concentrations into the flow cell. After eleven association-dissociation cycles, the hydrogel maintained its glucose sensitivity (Fig. 2e), thus showing the sensor has a good recoverability to glucose.

While glucose is predominant, human blood also contains other sugars such as fructose, galactose, sucrose and so on..$^{34-36}$ However, the effect of these sugars, even at such relatively low concentration $(<0.1 \mathrm{mM}$ (ref. 37-39)), may be to act as interferents to any glucose measurement using phenylboronic acids. To assess the influence of possible competitive binding of $0.1 \mathrm{mM}$ of other saccharides on the glucose, an experiment was carried out in which the sensor chip was exposed to $3.9 \mathrm{mM}$ glucose with and without $0.1 \mathrm{mM}$ of other saccharides. Fig. $2 \mathrm{f}$ shows that the presence of fructose, maltose and lactose lead to a slight increase in response frequency, compared to the presence of glucose alone. But QCM-based sensor is also able to detect physiologically relevant glucose concentrations also in the presence of other competing sugars within the error range. 


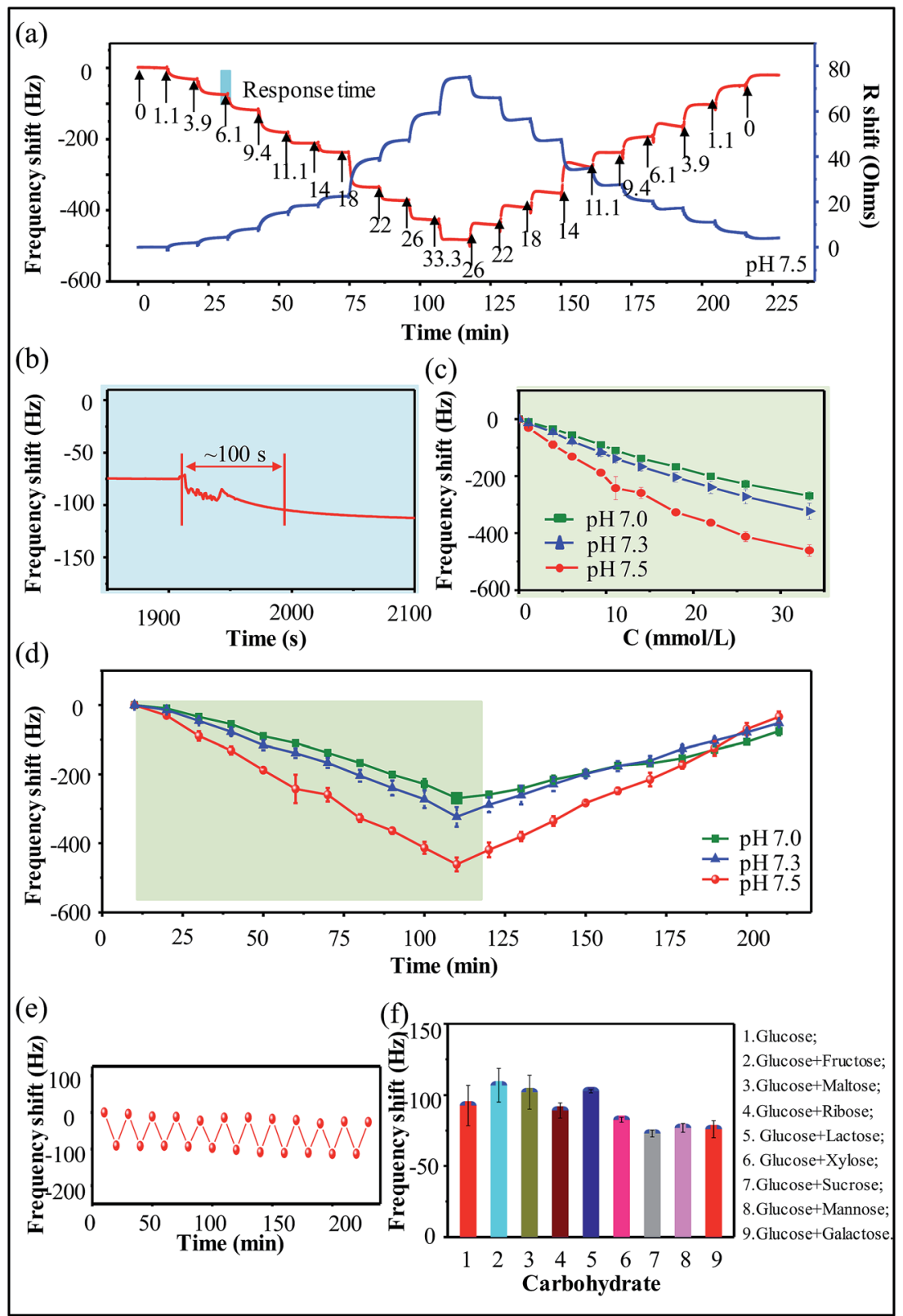

Fig. 2 Glucose sensing. (a) Frequency shift with varying glucose concentrations. (b) Response time. (c) Relationship between frequency shift and glucose concentration. (d) Glucose responses at different pH. (e) Reversibility. (f) Interference.

\section{Biocompatibility of the glucose sensors}

To assess the biocompatibility of the hydrogel sensor, we used microscopy to image L929 cells exposed to the coated chips. No significant difference was observed between cells in the experimental group and the control group (consisting of a reagent control and a negative control) (Fig. 3a). The experimental group cells exhibited normal cellular morphology, intact cellular membranes, and no exfoliation or dissolution; thus, there was no evidence of cytotoxicity. We also used the methylthiazolyl-tetrazolium (MTT) assay to evaluate the cytotoxicity of the hydrogel-coated chips, according to the national standard GB/T 16886-2001. ${ }^{40}$ MTT results showed that the relative increment rate (RGR) were greater than $90 \%$ for incubation periods of $24 \mathrm{~h}$ and $48 \mathrm{~h}$ (Fig. 3b). According to the GB/T 16175-1996, ${ }^{41}$ this is categorized as a grade 1 reaction. These results confirmed that the hydrogel-coated chips were not toxic to cells.

After subcutaneous implantation of the chips into SD rats, tissues that were in direct contact with the coated chips were imaged, and no difference was observed between the experimental group and the control group. Tissues were imaged at 7 days post-implantation (Fig. 3c). After surgery, all the SD rats' diet, activities and defecation were normal. We did not find the surgical incision of rats with pus and local tissue necrosis (Fig. S3†). At 7 days post-implantation, tissue morphology was normal, and there was no evidence of inflammatory cell infiltration into the tissue. No fibrous tissue twined around the 


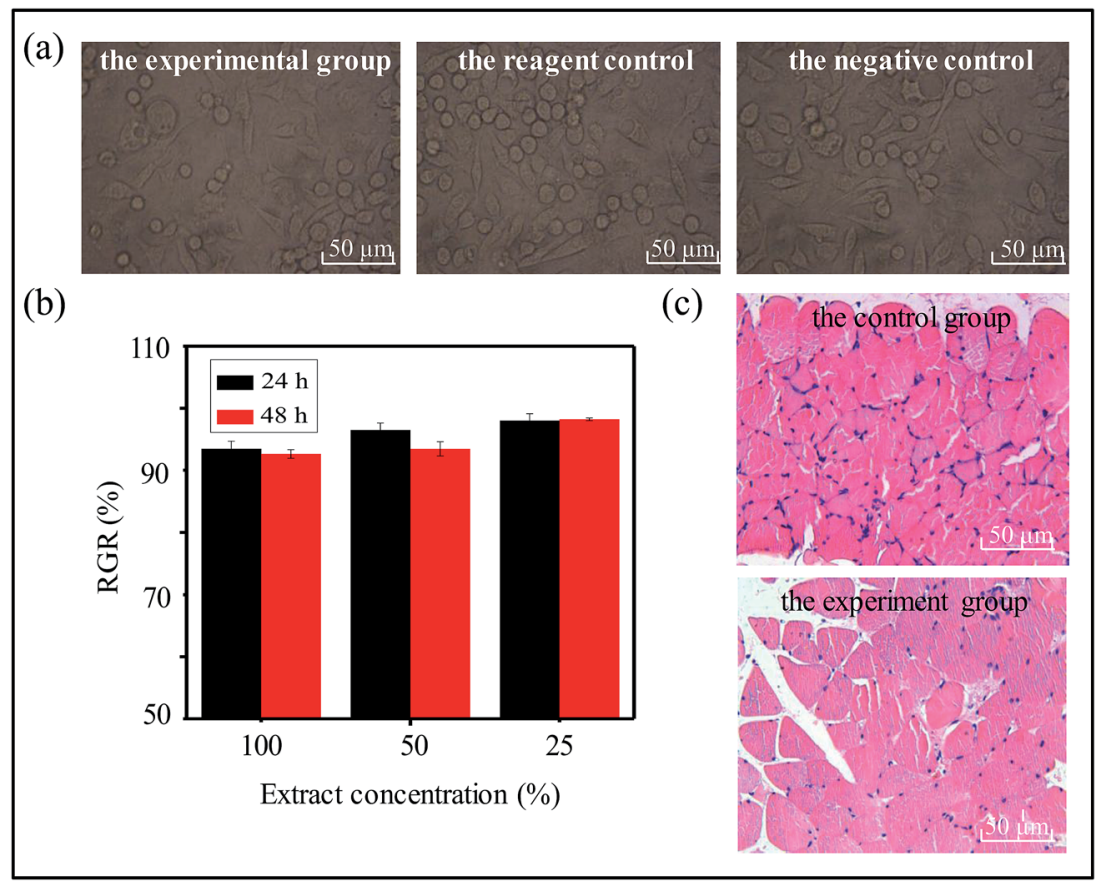

Fig. 3 Biocompatibility. (a) The microscopic images of the cells. (b) The RGR value of experiment and control group $24 \mathrm{~h}$ and $48 \mathrm{~h}$. (c) The animal tissues microscopic images of the control and experiment group at seven days after implantation.

implants and the hydrogels had not fallen off the gold-plate silicon with complete surface morphology.

\section{Conclusions}

As compared to GOx- and Con A-based glucose sensors, boronic acid-containing hydrogel exhibits favorable properties as an implantable glucose-sensitive material including stability, durability, long lifetime and low cost. In this study, we immobilized a glucose-sensitive hydrogel onto quartz crystals by means of surface-initiated polymerization, and employed the chip in QCM system for rapid glucose sensing. Our hydrogel displayed a response time ( $\sim 100 \mathrm{~s})$ as compared to existing boronic acid-hydrogel systems and full range BGC detection (1.1-33.3 mM) with typical physiological concentration in the interstitial fluid $\mathrm{pH}$ range (7.0-7.5). For potential application, hydrogel-coated quartz crystals could be implanted into subcutaneous tissue, the quartz resonance changes stimulated by glucose concentration fluctuations in vivo can be read through an external detection circuit, from which we could predict the BGC by formula conversion. In general, given its fast response and high sensitivity to glucose, durability and biocompatibility, this boronic acid-containing QCM system represents a promising implantable sensing platform for the continuous monitoring of glucose in diabetic patients.

\section{Experimental section}

Instruments and reagents

Instruments. Quartz crystal microbalance (QCM 200, Stanford Research Systems), Ultraviolet analyzer (ZF-20D, Shanghai Guanghao Ltd.), Spin coater (KW-4B, Beijing Teesside Case Electronics
Co., Ltd.). Ultrasonic cleaner (KQ 3200DE, Kunshan Ultrasonic Instruments Ltd), FTIR spectrometer (Nicolet iN10, Thermo Fisher Scientific Inc.), Atomic force microscope (Dimension Icon, Bruker), X-ray photoelectron spectroscopy (ESCALAB250Xi, Thermo Fisher Scientific Co., Ltd.), Paraffin embedding station (EG1150, Leica), Paraffin slicing machine (RM2235, Leica), Motic microscope (BA400, MOTIC Industrial Group Co., Ltd).

Reagents. 3-Aminopropyltriethoxysilane (APS, 98\%, Alfa Aesar), 3-acrylamidophenylboronic acid (3-APB, 98\%, Frontier Scientific), 2,2-dimethoxy-1,2-diphenyl-ethanone (DMPA, >98\%, Tokyo Chemical Industry), acrylamide (AM, 98.5\%, Xilong Chemical Industry), $N, N^{\prime}$-methylenebisacrylamide (BIS, 98\%, Sinopharm Chemical Reagent Ltd.), Analytically pure reagents (glucose, sodium phosphate dibasic dodecahydrate, potassium dihydrogen phosphate, maleic anhydride). Trypsin, L929 murine fiber cells and penicillinstreptomycin mixture $\left(100^{*}\right)$ were purchased from Beijing Prosperous Biological Technology Co., Ltd. Thirty-six SD rats (eighteen male and eighteen female), 220-240 g, were purchased from Beijing D Tong Lihua Experimental Animal Technical Co., Ltd.

For biocompatibility experiments, a gold layer with an approximate thickness of $100 \mathrm{~nm}$ was deposited onto monocrystalline silicon $(111)\left(0.6 \times 0.6 \mathrm{~cm}^{2}\right)$ by vacuum sputtering. An AT-cut quartz crystal chip with gold electrode, with a resonance frequency of $5 \mathrm{MHz}$ and diameter of $25 \mathrm{~mm}$, was used for the QCM test. And on the gold electrode was all investigated $\sim 250 \mathrm{~nm}$ hydrogel film.

\section{Synthesis of smart hydrogel}

Surface modification of quartz crystals. Gold-coated quartz crystals were sonicated for 10 minutes with piranha solution $\left(\mathrm{H}_{2} \mathrm{SO}_{4}\left(96 \%\right.\right.$ w/w) with $\mathrm{H}_{2} \mathrm{O}_{2}(30 \% \mathrm{w} / \mathrm{w})$ solutions in the volume 
ratio 7 : 3.). Processed quartz crystals were washed with redistilled water, then were placed in acetone, ethanol and redistilled water, respectively, each solution was sonicated for 10 minutes, in an ultrasonic cleaner. After the quartz crystals were dried with nitrogen, they were immersed in a mixed solution of 3-aminopropyltriethoxysilane and methylbenzene (v/v $1: 10)$ under nitrogen. Twelve hours later, the quartz crystals were rinsed with ethanol and subsequently dried with nitrogen. The dried quartz crystals were then immersed in a mixed solution of maleic anhydride and dimethylformamide (2\%) for twenty-four hours before being rinsed with ethanol and dried with nitrogen. ${ }^{42,43}$

Deposition of smart hydrogel films. First, we prepared a $5 \mathrm{~mol} \mathrm{~L}^{-1}$ pre-polymer solution consisting of $18 \% 3$-APB, $2 \%$ BIS, 78\% AM, and 2\% DMPA (by mass) in dimethyl sulfoxide as the solvent. Second, $30 \mu \mathrm{L}$ of pre-polymer solution was deposited onto the upper electrode of the quartz crystal and spincoated for one minute $(3500 \mathrm{rpm})$. Third, the coated quartz crystals were placed under ultraviolet irradiation $(\lambda=365 \mathrm{~nm})$ under nitrogen for $30 \mathrm{~min}$ for polymerization. Finally, the filmcoated quartz crystals were repeatedly rinsed with ethanol and redistilled water.

Instruction manual. We remained the waste piranha solution alone placed in the glass waste bottle, sealed with plastic film and opened a few small hole. Then placed bottle in a fume hood.

\section{Verification of glucose sensitivity}

A quartz crystal coated with a glucose-sensitive hydrogel film was dried with nitrogen and installed into the flow cell of the QCM 200 system. PBS $\left(0.1 \mathrm{~mol} \mathrm{~L}^{-1}\right)$ was pumped into the flow cell continuously, and the frequency of the crystal was monitored in real time using the QCM data acquisition software. After the frequency was stabilized (frequency shift $\leq \pm 2 \mathrm{~Hz}$ in $10 \mathrm{~min}$ ), we evaluated the glucose detection capacity of the material. Solutions $(3 \mathrm{~mL})$ of increasing and then decreasing glucose concentrations (from 0.0 to $33.3 \mathrm{mmol} \mathrm{L}^{-1}$ with PBS, and then in reverse) were pumped into the flow cell every $10 \mathrm{~min}$, and the frequency shift $\Delta F$ was recorded for each glucose concentration. Several cycles of this experiment were conducted under different $\mathrm{pH}$ levels from 7.0 to 7.5 to mimic the human interstitial fluid environment. Further experiments were conducted to verify the reversibility of the film by repeatedly pumping glucose solutions of 0.0 and $3.9 \mathrm{mmol} \mathrm{L}^{-1}$ concentrations into the flow cell.

\section{Biological experiment}

All animal experiments were performed complying with the National Institutes of health (NIH) guidelines for the care and use of laboratory animals of National Center for Nanoscience and Technology Animal Study Committee's requirements and according to the protocol approved by the Institutional Animal Care.

\section{Cell toxicity}

(1) Quantitative evaluation. The culture medium used in the experiment was the dulbecco's minimum essential medium
(DMEM) culture medium containing $10 \%$ fetal bovine serum (FBS) and 1\% penicillin-streptomycin. The detailed operational procedures for the MTT assay are as follows: (1) Extraction: each chip was soaked in $4 \mathrm{~mL}$ of culture medium and extracted at $37{ }^{\circ} \mathrm{C}$ for $24 \mathrm{~h}$. The same conditions were treated with negative contrast (high density polyethylene) and reagent contrast (under the condition of without test materials, according to the extraction conditions and test steps to get the extraction medium). (2) Cell inoculation: a single cell suspension was prepared in culture medium, and 5000 cells were seeded in 96 well plates with a volume of $200 \mu \mathrm{L}$ per well. (3) Extraction solution treatment: culture medium was removed after cells fully adhered to the plate wall, and $200 \mu \mathrm{L}$ of leaching solution was added to each well. The different concentration leaching solution $(25 \%, 50 \%$ or $100 \%)$ was obtained by diluting the culture solution. Cells were cultured $24 \mathrm{~h}$ and $48 \mathrm{~h}$ after the addition of the extraction solution. For all experiments, three replicate chips were used for each data point, and six parallel wells were used for each dilution. (4) Color reaction: MTT was dissolved in PBS (pH 7.4), and $20 \mu \mathrm{L}$ (5 mg mL ${ }^{-1}$ ) was added to each well. (5) Termination reaction: cells were incubated in MTT for $4 \mathrm{~h}$, subsequently, the supernatant was removed and $150 \mu \mathrm{L}$ of dimethyl sulfoxide was added to each well to dissolve the crystals, and the plate was placed on the shaker with low-speed oscillation. (6) Colorimetric detection: the absorbance was detected by the enzyme-linked immune sorbent assay (ELISA) at a wavelength of $492 \mathrm{~nm}$ wavelength, and the relative increment rate $\left(\mathrm{RGR}=\left(A_{\text {experiment }} / A_{\text {reagent }}\right) \times 100 \%\right)$ were calculated. For quantitative evaluation of cytotoxicity, the following metrics were used: level $0, \geq 100 \%$ or higher; level 1, 7599\%; level 2, 50-74\%; level 3, 25-49\%; level 4, 1-24\%, level 5, 0.

(2) Qualitative evaluation. Cells were seeded in 6-well plates and were cultured under $5 \% \mathrm{CO}_{2}$ at $37{ }^{\circ} \mathrm{C}$ in the incubator. After the cells had adhered to the plate, they were incubated in the extracted solution for $24 \mathrm{~h}$ and were then evaluated by microscopy for cell morphology, vacuolization, loss, cell dissolved, and membrane integrity, and were scoring accordingly. The scoring method was as follows: 0 , no cell toxicity; 1 , mild cytotoxicity; 2 , moderate cytotoxicity; 3 , severe cytotoxicity.

\section{Implant experiment}

Test samples and control samples (high-density Teflon) were implanted subcutaneously in the backs of rats. A total of 12 rats were used (six male, six female), and each group receiving implants for 7 days. After implantation, rats were observed and evaluated daily. After 7 implantation period, twelve rats were sacrificed to visualize the implant and the surrounding tissues. Paraffin sectioning, HE staining, and light microscopy were performed to evaluate the degree of tissue reaction. Additionally, the surface morphology of the chip was evaluated for any changes post-implantation. Chips that exhibited any changes in morphology, film position, decomposition, or extensive presence of biomass were considered to fail the implantation test.

\section{Conflicts of interest}

There are no conflicts to declare. 


\section{Acknowledgements}

This work was supported by the National Basic Research Program of China (Grant No. 2015CB932400, 2016YFA0201600), the National Natural Science Foundation of China (Grant No. $51372045,11504063)$, the Bureau of International Cooperation, Chinese Academy of Science (121D11KYSB20130013), and the key program of the bureau of Frontier Sciences and Eduction Chinese Academy of Sciences (QYZDB-SSW-SLH021).

\section{Notes and references}

1 B. Kovatchev, L. Heinemann, S. Anderson and W. Clarke, Diabetes Care, 2008, 31, 1160-1164.

2 T. S. Bailey, H. C. Zisser and S. K. Garg, Diabetes Technol. Ther., 2007, 9, 203-210.

3 I. M. Wentholt, J. B. Hoekstra, M. A. Vollebregt, J. Hans Devries and A. A. Hart, Diabetes Care, 2005, 28, 2871-2876.

4 G. McGarraugh, Diabetes Technol. Ther., 2009, 11, S17-S24.

5 M. V. Voinova, M. Jonson and B. Kasemo, Biosens. Bioelectron., 2002, 17, 835-841.

6 M. Rodahl, F. Höök and B. Kasemo, Anal. Chem., 1996, 68, 2219-2227.

7 M. Rodahl and B. Kasemo, Sens. Actuators, B, 1996, 37, 111116.

8 X.-L. Su and Y. Li, Biosens. Bioelectron., 2005, 21, 840-848.

9 R. Hao, D. Wang, X. Zhang, G. Zuo, H. Wei, R. Yang, Z. Zhang, Z. Cheng, Y. Guo, Z. Cui and Y. Zhou, Biosens. Bioelectron., 2009, 24, 1330-1335.

10 F. R. Kaufman, J. Austin, A. Neinstein, L. Jeng, M. Halvorson, D. J. Devoe and P. Pitukcheewanont, J. Pediatr., 2002, 141, 625-630.

11 D. C. Klonoff, Diabetes Care, 2005, 28, 1231-1239.

12 H.-C. Wang, H. Zhou, B. Chen, P. M. Mendes, J. S. Fossey, T. D. James and Yi-T. Long, Analyst, 2013, 138, 7146-7151.

13 K. Ngamdee, T. Noipa, S. Martwiset and T. Tuntulani, Sens. Actuators, B, 2011, 160, 129-138.

14 C. Shimpuku, R. Ozawa, A. Sasaki, F. Sato, T. Hashimoto, A. Yamauchi, I. Suzuk and T. Hayashita, Chem. Commun., 2009, 13, 1709-1711.

15 A. P. Davis and R. S. Wareham, Angew. Chem., 1999, 38, 2978-2996.

16 S. L. Wiskur, H. Aithaddou and J. J. Lavigne, Acc. Chem. Res., 2001, 34, 963-972.

17 Y. Guan and Y. Zhang, Chem. Soc. Rev., 2013, 42, 8106-8121.

18 J. N. Cambre and B. S. Sumerlin, Polymer, 2011, 52, 46314643.

19 K. Lacina, P. Skládal and T. D. James, Chem. Cent. J., 2014, 8, 60-77.

20 C. C. Deng, W. L. A. Brooks, K. A. Abboud and B. S. Sumerlin, ACS Macro Lett., 2015, 4, 220-224.
21 X. Jin, X. Zhang, Z. Wu, D. Teng, X. Zhang, Y. Wang, Z. Wang and C. Li, Biomacromolecules, 2009, 10, 1337-1345.

22 C. Sugnaux and H.-A. Klok, Macromol. Rapid Commun., 2014, 35, 1402-1407.

23 C. Zhang, G. G. Cano and P. V. Braun, Adv. Mater., 2014, 26, 5678-5683.

24 G. Ye and X. Wang, Biosens. Bioelectron., 2010, 26, 772-777.

25 J. T. Suri, D. B. Cordes, F. E. Cappuccio, R. A. Wessling and B. Singaram, Angew. Chem., Int. Ed., 2003, 42, 5857-5859.

26 D. Shno, A. Kubo, Y. Murata, Y. Koyama, K. Kataoka, A. Kikuchi, Y. Sakurai and T. Okano, J. Biomater. Sci., Polym. Ed., 1996, 7, 697-705.

27 C. Ancla, V. Lapeyre, I. Gosse, B. Catargi and V. Ravaine, Langmuir, 2011, 27, 12693-12701.

28 I. Hisamitsu, K. Kataoka, T. Okano and Y. Sakurai, Pharm. Res., 1997, 14, 289-293.

29 K. Kataoka, H. Miyazaki, M. Bunya, T. Okano and Y. Sakurai, J. Am. Chem. Soc., 1998, 120, 12694-12695.

30 R. Gabai, N. Sallacan, V. Chegel, T. Bourenko, E. Katz and I. Willner, J. Phys. Chem. B, 2001, 105, 8196-8202.

31 A. M. Horgan, A. J. Marshall, S. J. Kew, K. E. S. Dean, C. D. Creasey and S. Kabilan, Biosens. Bioelectron., 2006, 21, 1838-1845.

32 V. L. Alexeev, A. C. Sharma, A. V. Goponenko, S. Das, I. K. Lednev, C. S. wilcox, D. N. Finegold and S. A. Asher, Anal. Chem., 2003, 75, 2316-2323.

33 Y. J. Huang, W. J. Ouyang, X. Wu, Z. Li, J. S. Fossey, T. D. James and Y.-B. Jiang, J. Am. Chem. Soc., 2013, 135, 1700-1703.

34 S. Tierney, S. Volden and B. T. Stokke, Biosens. Bioelectron., 2009, 24, 2034-2039.

35 G. Ye and X. Wang, Biosens. Bioelectron., 2010, 26, 772-777.

36 M.-C. Lee, S. Kabilan, A. Hussain, X. Yang, J. Blyth and

C. R. Lowe, Anal. Chem., 2004, 76, 5748-5755.

37 A. M. Horgan, A. J. Marshall, S. J. Kew, K. E. S. Dean, C. D. Creasey and S. Kabilan, Biosens. Bioelectron., 2006, 21, 1838-1845.

38 T. D. James, K. R. A. Samankumara Sandanayake and S. Shinkai, Angew. Chem., Int. Ed. Engl., 1994, 33, 2207-2209.

39 H. Eggert, J. Frederiksen, C. Morin and J. C. Norrild, J. Org. Chem., 1999, 64, 3846-3852.

40 State Administration of Quality Supervision, Inspection and Quarantine of the People's Republic of China, GB/T16886, Standards Press of China, Beijing, 2001.

41 National Technical Supervision Bureau, GB/T 16175, Standards Press of China, Beijing, 1996.

42 M. Lazerges, H. Perrot, N. Rabehagasoa and C. Compère, Biosensors, 2012, 2, 245-254.

43 P. Vejayakumaran, I. A. Rahman, C. S. Sipaut, J. Ismail and C. K. Chee, J. Colloid Interface Sci., 2008, 328, 81-91. 